\title{
An Oligonucleotide Microarray Bait for Isolation of Target Gene Fragments
}

\author{
Shi Rong, Ma Wen-li*, Liu Cui-hua, Song Yan-bin, Mao Xiang-ming and Zheng Wen-ling* \\ Institute of Molecular Biology, First Military Medical University, Guangzhou, 510515, P.R. China \\ Institute of Molecular Oncology, Liu Hua Qiao Hospital, Guangzhou, 510010, P.R. China
}

Received 15 May 2003, Accepted 18 June 2003

\begin{abstract}
A new molecular-baiting method was studied by retrieving targeted gene fragments from an oligonucleotide microarray bait after hybridization. To make the microarray bait, 70-mer oligonucleotides that were designed to specifically represent the SSA1 gene of Saccharomyces cerevisiae were printed on the slide. Samples of the Saccharomyces cerevisiae mRNA were extracted and labeled by the RD-PCR (Restriction Display PCR) method using the Cy5-labelled universal primer, then applied for hybridization. The sample fragments that hybridized to the microarray were stripped, and the eluted cDNAs were retrieved and cloned into the pMD 18-T vector for transformation, plasmid preparation, and sequencing. BLAST searching of the GenBank database identified the retrieved fragments as being identical to the SSA1 gene (from 2057-2541bp). A new method is being established that can retrieve the sample fragments using an oligo-microarray-bait.
\end{abstract}

Keywords: Fluorescence labeling, Microarray stripping, Oligo-microarray-bait, RD-PCR

\section{Introduction}

Although the method of isolating the target genes by constructing a cDNA library is widely used, it is a tedious procedure. In this report, we used a specific long oligonucleotide microarray bait which can isolate the target gene by hybridization. In order to retrieve the targeted gene fragments and verify our experimental design, we also used the RD-PCR labeling method (Shi et al., 2003) to add an adaptor to both sides of the sample fragments. All of the

*To whom correspondence should be addressed.

Tel: 86-20-6164-8210; Fax: 86-20-6164-7755

E-mail: wenli@fimmu.edu.cn fragments that were stripped down from the array can then be retrieved by PCR using the universal primer that matched the adaptor. With a universal tag attached, it is also easier to verify the target gene from the experiments. With this model system, we can acquire the target gene directly from the RD fragments without the necessity of building a cDNA library. We can also evaluate the specificity and stringency of the microarray probes by these fragments and use them for further research of the sample.

\section{Materials and Methods}

Saccharomyces cerevisiae and E. coli JM 109 were provided by the Liu Hua Qiao Hospital Medical Center. The YPD and LB culture medium were obtained from OXOID Inc (Ogdensburg, USA). The QuickPrep mRNA purification kit and cDNA synthesize kit were purchased from Amersham Pharmacia Biosciences (Piscataway, USA). The restriction enzyme Sau3AI, Taq DNA polymerase, T4 DNA ligase, pMD 18-T Vector were bought from TaKaRa Bio Inc (Shiga, Japan). The universal primer (5' GTTTGGCTGGTGTGGA TC 3'), Cy5 labeled universal primer (5'Cy5-UTTGGCTGGTGTG GATC 3') and adaptors (primer SIP: 5'pGATC ${ }^{\mathrm{m}}$ CACACCAGCCA AACCCA3'; SIR: 5GGTTTGGCTGGTGTG3') were synthesized by BIOASIA Inc (Taipei, Taiwan). The other reagents were as follows: QIAquick PCR product purification kit (QIAGEN, Hilden, Germany)), DEPC and Poly-L-Lysine (Sigma, St. Louis, USA), silanlized slides (DAKO, Carpinteria, USA), DMSO and Formamide (Amresco, Solon, USA), 70-mer oligo yeast sample set (QIAGEN), succinic anhydride (Aldrich, Milwaukee, USA), 1methyl-2-pyrrolidinone (Aldrich), and sodium borate (Merck, Schuchardt, Germany).

Methods The total RNAs of Saccharomyces cerevisiae were extracted by a Phenol/Freeze protocol (Schmitt et al., 1990). The mRNAs were purified using a QuickPrep mRNA purification kit and were reverse transcripted into the double-stranded cDNAs using a cDNA synthesize kit, according to the manufacturers' instructions. Concentrations of the cDNAs were measured in a 
Beckman DU530 UV spectrometer.

The ds cDNAs were digested by the restriction enzyme Sau3AI for about $4 \mathrm{~h}$. On both sides of the digested fragments there were cohesive ends of GATC. The two primers, SIP $(500 \mu \mathrm{g} / \mathrm{mL})$ and SIR $(600 \mu \mathrm{g} / \mathrm{mL})$, were heated to $90^{\circ} \mathrm{C}$ for $5 \mathrm{~min}$, then gradually cooled down to $20^{\circ} \mathrm{C}$ in 30 min for annealing in order to obtain the double-stranded universal adaptors. The adaptors were linked to both sides of the digested fragments by the T4 DNA ligase so that all of these fragments with adaptors could be amplified and labeled by the RD-PCR method. The PCR reactions contained $25 \mu \mathrm{L}$ of $2 \times \mathrm{PCR}$ buffer $(100 \mathrm{mmol} / \mathrm{l} \mathrm{KCl}, 20 \mathrm{mmol} / \mathrm{l}$ Tris- $\mathrm{HCl} \mathrm{pH} 8.3$, $3 \mathrm{mmol} / \mathrm{l} \mathrm{MgCl}_{2}$ ), $1 \mu \mathrm{l}$ of cDNA fragments, $5 \mu \mathrm{l}$ of Cy5 labeled universal primer, $18 \mu \mathrm{l}$ of $\mathrm{ddH}_{2} \mathrm{O}$, and $1 \mu \mathrm{l}$ of Taq DNA polymerase. The PCR amplifications were performed in an ABI PCR System 2700 with an initial denaturing temperature of $95^{\circ} \mathrm{C}$ for $5 \mathrm{~min}$. This was followed by 25 cycles of $30 \mathrm{~s}$ at $95^{\circ} \mathrm{C}, 30 \mathrm{~s}$ at $60^{\circ} \mathrm{C}, 1 \mathrm{~min}$ at $72^{\circ} \mathrm{C}$, and a final extension of $5 \mathrm{~min}$ at $72^{\circ} \mathrm{C}$.

The slide was coated by Poly-L-Lysine according to the protocol (Brown, 1999) of Pat Brown's lab at Stanford University. The 70mer oligo sample set was spun down in a plate centrifuge and resuspended into $12.5 \mu \mathrm{l}$ of $50 \%$ DMSO to reach a final concentration of $1 \mu \mathrm{g} / \mu \mathrm{l}$. The plate was then resealed and placed on an orbital shaker overnight at $4^{\circ} \mathrm{C}, 60 \mathrm{rpm}$. One oligo fragment, which represented the SSA1 gene of yeast (GenBank Accession: YAL005C), was selected and printed into a $18 \times 18$ microarray on the slide using the Cartesian PixSys 5500 robot. The slide was then re-hybridized and snap-dried in a plate at $140^{\circ} \mathrm{C}$. A BIO-RAD UV Cross-linker was used to immobilize the DNAs onto the slide with $65 \mathrm{~mJ}$ of energy. The slide was treated with the blocking solution (335 ml 1-methyl-2-pyrrolidinone, $6 \mathrm{~g}$ succinic anhydride and $15 \mathrm{ml} 1 \mathrm{M} \mathrm{NaBorate}, \mathrm{pH} 8$ ) and stored for later use.

The PCR labeled sample was purified using a PCR product purification kit, dissolved in a hybridization buffer $(25 \%$ formamide, $5 \times \mathrm{SSC}, 0.1 \% \mathrm{SDS}$ ), denatured for $5 \mathrm{~min}$ at $95^{\circ} \mathrm{C}$, and spun for $2 \mathrm{~min}$ at the maximum speed to cool down. The slide was placed in a Corning CMT-Hybridization chamber, then $6 \mu \mathrm{l}$ of the sample were pipetted onto the array. A silicone pre-treated coverslip

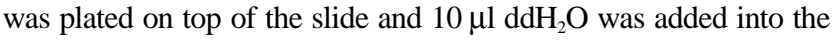
two holes at both ends of the hybridization chamber to maintain the humidity. Next, the hybridization chamber was sealed and submerged in a $42^{\circ} \mathrm{C}$ water-bath for $16 \mathrm{~h}$.
The hybridization chamber was disassembled right side up. The coverslip was removed by immersing the slide in the washing solution I $\left(2 \times \mathrm{SSC}, 0.1 \% \mathrm{SDS}, 42^{\circ} \mathrm{C}\right)$ for $5 \mathrm{~min}$. The slide was then transferred into the washing solution II $(0.1 \times \mathrm{SSC}, 0.1 \% \mathrm{SDS}$, room temperature) for $10 \mathrm{~min}$, then placed in the washing solution III $(0.1 \times \mathrm{SSC}$, room temperature) 5 times, 1 min each. During the wash steps, the jar where the slide was placed was put onto an orbit shaker $(40 \mathrm{rpm})$ to make sure that the fragments of the nonspecific hybridization were thoroughly washed out. Finally, the slide was rinsed in $\mathrm{ddH}_{2} \mathrm{O}$ and $100 \% \mathrm{EtOH}$, and spun to dry.

The slide was scanned using a Packard ScanArray Lite microarray scanner, under the conditions of $90 \%$ laser power and $72 \%$ PMT.

The hybridized array was stripped by $30 \mu \mathrm{l}$ of $0.01 \mathrm{M} \mathrm{NaOH}$ for $10 \mathrm{~min}$ at $42^{\circ} \mathrm{C}$. During this process, the array was gently pipetted to ensure the stripping. Next, the slide was scanned under the same conditions to check the stripping efficiency. The process was repeated once more to ensure that all of the sample fragments were washed from the array. The retrieved solution was neutralized by $60 \mu \mathrm{l} 0.01 \mathrm{M} \mathrm{HCl}$ and vacuum-dried by a Savant Integrated SpeedVac System Iss110. The pellet was dissolved in $5 \mu \mathrm{l}$ of $\mathrm{ddH}_{2} \mathrm{O}$, which was used as a template of a new RD-PCR amplification by the universal primer without fluoresce. The product was identified by agarose gel electrophoresis.

The PCR product was inserted overnight into the pMD 18-T vector, then the recombined plasmids were transfected into the $\mathrm{E}$ coli. Strain JM109 and cultured in the solid LB culture medium with X-Gal, IPTG, and Amp. Thirty positive colonies were chosen for the enlarged culture in a liquid LB culture medium, and identified by a PCR amplification and agarose gel electrophoresis.

\section{Results}

Figure 1a shows the hybridization results of the yeast cDNA fragments with the oligo microarray under the condition of $90 \%$ laser energy and $72 \%$ PMT. Figures $1 \mathrm{~b}$ and $1 \mathrm{c}$ show the scan results after the first and second stripping steps under the same conditions. From the figures, we discover that after the two stripping steps, all of the hybridized fragments were
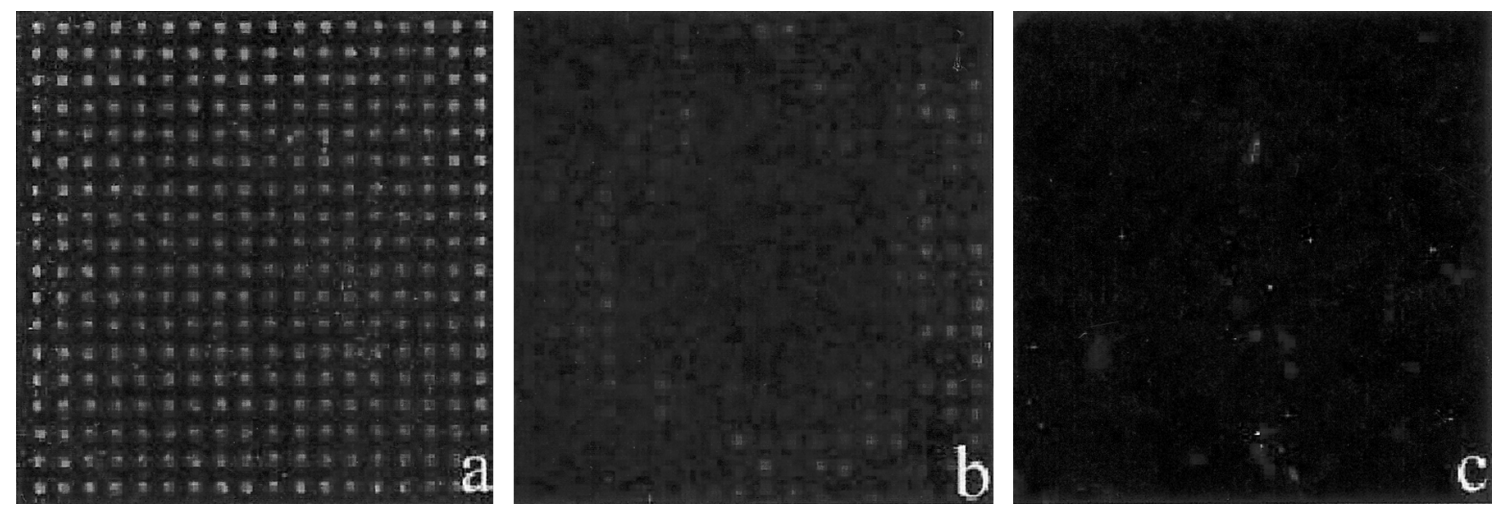

Fig. 1. Result of hybridization before and after the stripping processes. a, immediately after the completion of hybridization with the yeast cDNA fragments; $b$, scanned results after the 1st round of stripping; $c$, scanned results after the 2nd stripping process. 

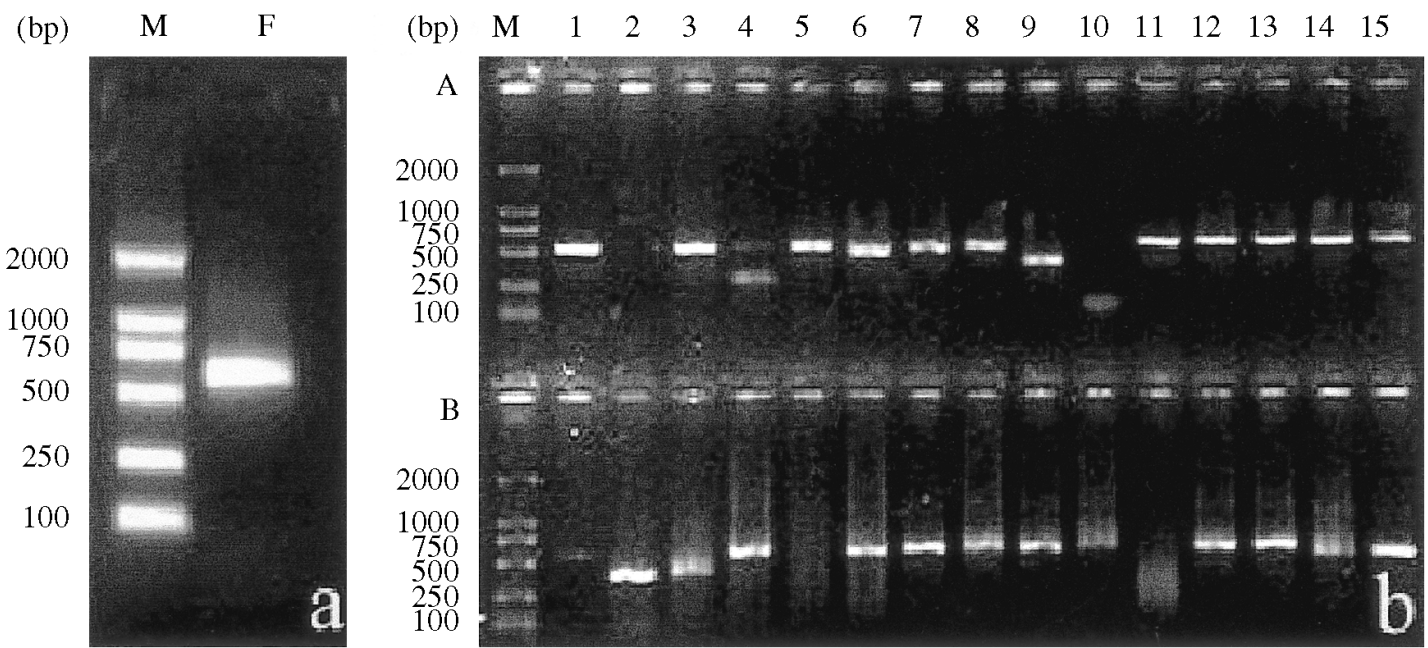

Fig. 2. (a) Agarose gel electrophoresis of retrieved fragment. M, MDL2000 standard DNA ladder; F, retrieved fragment. (b) Agarose gel electrophoresis of cology PCR products of positive colonies. M, MDL2000 standard DNA ladder, A1-A15 and B1-B15, PCR products of positive clones.

washed off the microarray as there was no fluorescence signal after the second stripping.

Figure 2a shows the agarose gel electrophoresis of the PCR product by using the retrieved fragments as templates. The fragment is a single band of about $600 \mathrm{bp}$. Thirty transformed positive $E$. coli colonies were picked out and colony PCR was conducted by the universal primer. Figure $2 b$ shows the agarose gel electrophoresis results of the colony PCRs identification. Most of the fragments were between 500 bp$750 \mathrm{bp}$, which appears to denote the retrieved fragment, as in Fig. 2a.

All of the positive colonies with an obvious band were selected for sequencing. These included A1, A3, A5, A6, A7, A8, A9, A11, A12, A13, A14, A15, B2, B4, B6, B7, B8, B9, $\mathrm{B} 12, \mathrm{~B} 13$, and B15. The BLAST search of the GenBank database showed that A1, A3, A5, A6, A7, A8, A11, A12, A13, A14, A15, B7, B8, B9, B12, and B13 were all the same fragments that we designated with universal adapter sequence tagging the end of the target cDNA fragment. A6, B4, B6, and B15 were sequences from the vector while A9 and B2 were fragments of $E$. coli. The sequencing and BLAST result of the A12 clone are shown in Fig. 3. The fragment belongs to the SSA1 protein and tRNA-Pro gene, which is a member of the $70 \mathrm{kD}$ heat shock protein family.

\section{Discussion}

With the rapid progress in the DNA synthesizing method, longer oligonucleotide microarray ( $\mathrm{Li}$ and Stormo, 2001) technology has recently been developed. This technology uses a 70-mer oligonucleotide that has been calculated to be the optimized length to represent genes of interest and facilitate efficient hybridization dynamics. The 70-mer oligo microarray, representing bona fide functional genes, should have a more homogeneous $T_{m}$ value than the cDNA fragments, whose length and GC content range widely. However, most of the advantages are in theory or have either more or less indirect evidence. Can an oligonucleotide probe of only 50-120 bases really represent a single gene? This concern needs to be corroborated by experimental studies. We apply here a new labeling method that in theory allows the retrieval of the target fragments that hybridized. This should provide a novel method of cDNA retrieval by using oligo microarray bait that is directly from the RD fragments instead of constructing a cDNA library. At the same time, it provides direct evidence for the verification of the quality of hybridization, such as specificity, hybridization stringency, stability, and reproducibility.

RD-PCR was previously a gene-separating method for microarray probe preparation (Bao et al., 2002; Zhang et al., 2002; Zhu et al., 2002); however, we found recently that it also had advantages in sample labeling. Since each cDNA has an adaptor on both sides, the sample can be labeled by a fluorescent universal primer through PCR amplification. This means that the fluorescent intensity we obtained from the scanner was determined by the quantity of the molecules that hybridized with the probes on the microarray. This eliminated the influence of the length of the sample fragments, in which longer fragments could incorporate more fluorescent-dNTP and consequently imprecisely emitted more intense hybridization signals. We can also retrieve the fragment by the PCR method with the known adaptors sequence to be used as amplification primers. In this study, we stripped the sample fragments from the microarray, and cloned them into a pMD 18 -T vector. BLAST searching of the GenBank database showed that the fragments that were derived to SSA1 were from 2057-2541. SSA1 is one of the members of the heat shock protein family. Our results not only corroborated the prediction that 70-mer oligonucleotide could correctly 


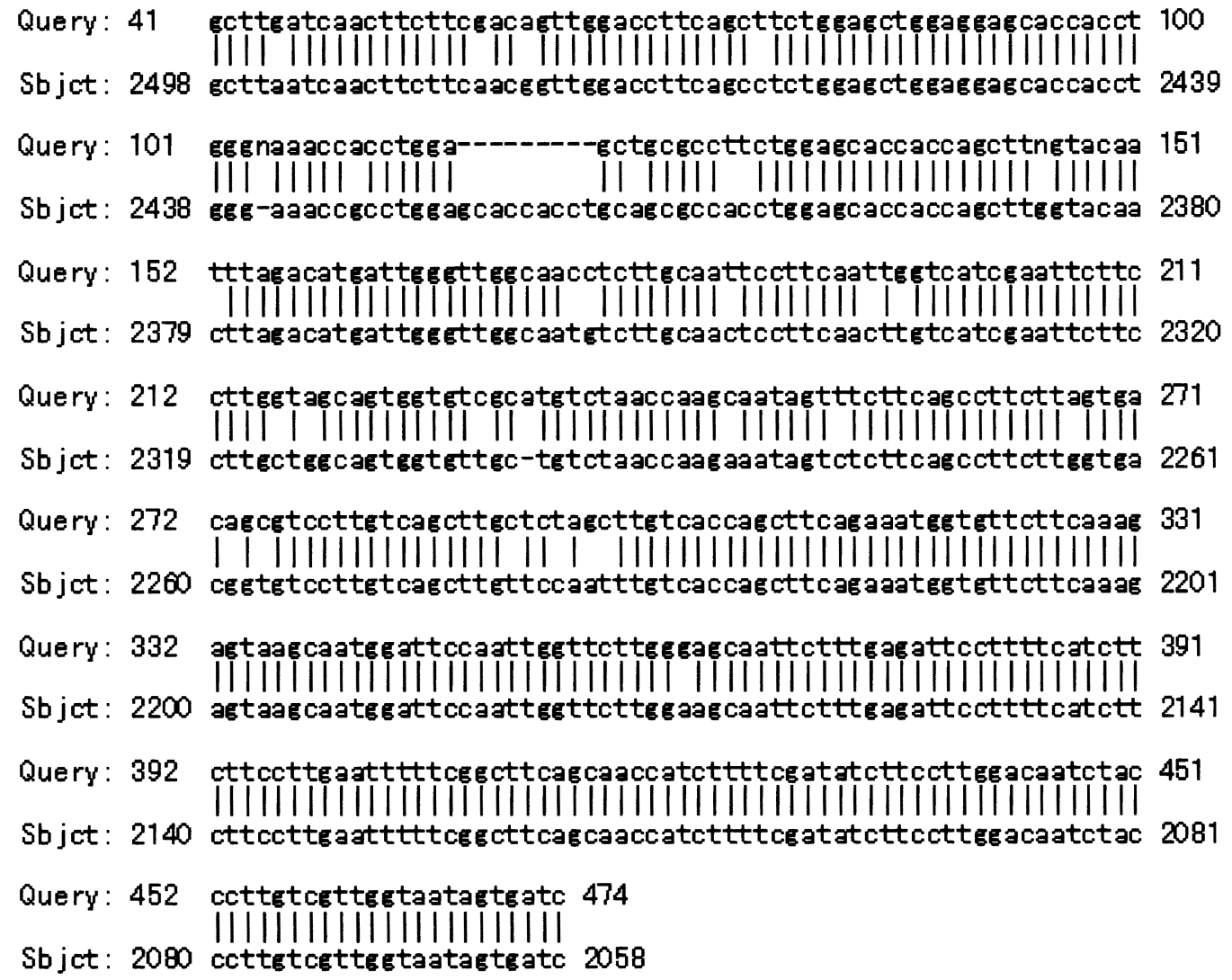

Fig. 3. BLAST searching results of clone A12. Identities of the fragment with the SSA1 gene (GenBank Accession: gi|312351|emb|X12926.1|SCSSA1) were 404/443 (91\%) and the gaps were 11/443 (2\%).

represent a gene or gene fragments to be used in microarray preparation, but also confirmed our experimental design, which developed an efficient way of retrieving target gene fragments by using an oligo microarray bait.

The immobilization ratio is one of the important factors that affects the quality of the microarray, especially for the oligo probe microarray when the probe strand was short. Many methods have been applied to improve the ratio, such as increasing the concentration of the probes, modification of the oligonucleotide (Raddatz et al., 2002), and improving the materials for coating (Benters et al., 2001; Consolandi et al., 2002) so that the oligo could attach to the substrates by a more stable covalent bond. For example, slide coating with the epoxy or aldehyde groups can form a covalent bond with the amine groups on the nucleic acid bases, especially when the oligonucleotide is amino modified (Zou et al., 2001). Nevertheless, the immobilization ratio of Poly-L-Lysin is relatively high when the probe concentration is increased, so it is still widely used in making an oligo microarray. Other materials (such as nylon membrane, plastic, or polymethyl methacrylate substrates) can sometimes be reused because they combine more tightly with the probes. The $\mathrm{NaOH}$ concentration in the stripping solution is critical, since the probes will be striped with the sample fragments if it is too high, but sample fragments will not be washed out completely if it is too low.

In conclusion, we established a labeling method for the quality control of microarray hybridization, which at the same time allows the retrieval of the sample fragment directly from the microarray. This oligo microarray bait method can also be used to acquire the targeted differentially expressed gene fragments, selecting the gene fragments which are represented by the oligos, and re-array them into a new microarray for diagnosis or other purposes. Compared with the conventional cDNA library screening techniques, this method will be more effective and efficient.

\section{References}

Bao, Z., Wenli, M., Qinghua, W., Qiuye, G., Yanbin, S. and Wenling, Z. (2002) Construction of a cDNA fragment library from SH-SY5Y cells using restriction display PCR. $B r . J$. Biomed. Sci. 59, 35-37.

Benters, R., Niemeyer, C. M. and Wohrle, D. (2001) Dendrimeractivated solid supports for nucleic acid and protein microarrays. Chembiochem. 2, 686-694.

Brown, P. O. (1999) http://cmgm.stanford.edu/pbrown/protocols/ 1_slides.html.

Consolandi, C., Castiglioni, B., Bordoni, R., Busti, E., Battaglia, C., Bernardi, L. R. and De Bellis, G. (2002) Two efficient 
polymeric chemical platforms for oligonucleotide microarray preparation. Nucleosides Nucleotides Nucleic Acids 21, 561580.

Li, F. and Stormo, G. D. (2001) Selection of optimal DNA oligos for gene expression arrays. Bioinformatics 17, 1067-1076.

Raddatz, S., Mueller-Ibeler, J., Kluge, J., Wass, L., Burdinski, G., Havens, J. R., Onofrey, T. J., Wang, D. and Schweitzer, M. (2002) Hydrazide oligonucleotides: new chemical modification for chip array attachment and conjugation. Nucleic Acids Res. 30, 4793-4802.

Schmitt, M. E., Brown, T. A. and Trumpower, B. L. (1990) A rapid and simple method for preparation of RNA from Saccharomyces cerevisiae. Nucleic Acids Res. 18, 3091-3092.

Shi, R., Ma, W. L., Song, Y. B., Li, L., Liu, C. H. and Zheng, W.
L. (2003) Two restriction fluorescence labeling methods for enhancing the signal-to-noise ratio of cDNA microarray hybridization. Di Yi Jun Yi Da Xue Xие Bao. 23, 124-126.

Zhang, B., Ma, W. L., Hu, Z. Y., Shi, R., Song, Y. B. and Zheng, W. L. (2002) A method for evaluation of the quality of DNA microarray spots. J. Biochem. Mol. Biol. 35, 532-535.

Zhu, J., Ma, W. L., Li, L., Yao, R. H. and Zheng, W. L. (2002) A method for construction of restriction cDNA library. Hereditas (Beijing) 24, 174-176.

Zou, Z. L., Wang, S. Q. and Wang, Z. Q. (2001) Preparation optimization and properties of the aldehyde microscopic slides for oligonucleotide microarray fabrication. Sheng Wu Gong Cheng Xие Bao. 17, 498-502. 